\title{
CLASSIFICATION OF TOTALLY UMBILICAL SUBMANIFOLDS IN SYMMETRIC SPACES
}

\author{
BANG-YEN CHEN
}

(Received 31 January 1979; revised 28 April 1980)

Communicated by J. Virsik

\begin{abstract}
A submanifold of a Riemannian manifold is called a totally umbilical submanifold if the second fundamental form is proportional to the first fundamental form. In this paper, we shall prove that there is no totally umbilical submanifold of codimension less than rank $M-1$ in any irreducible symmetric space $M$. Totally umbilical submanifolds of higher codimensions in a symmetric space are also studied. Some classification theorems of such submanifolds are obtained.
\end{abstract}

1980. Mathematics subject classification (Amer. Math. Soc.): 53 B 20, 53 C 35, 53 C 40.

\section{Introduction}

Let $N$ be an $n$-dimensional submanifold of an $m$-dimensional Riemannian manifold $M(n \geqslant 2)$ with metric $g$. Let $\nabla$ and $\tilde{\nabla}$ be the covariant differentiations on $N$ and $M$, respectively. Then the second fundamental form $h$ of the immersion is defined by the equation

$$
h(X, Y)=\tilde{\nabla}_{X} Y-\nabla_{X} Y,
$$

where $X$ and $Y$ are vector fields tangent to $N$. The submanifold $N$ is said to be totally umbilical if

$$
h(X, Y)=g(X, Y) \cdot H,
$$

for all vector fields $X, Y$ tangent to $N$, where $H=1 / n$ trace $h$ is the mean curvature vector of $N$ in $M$. The length of $H$ is called the mean curvature of $N$ in $M$. A totally umbilical submanifold with vanishing mean curvature is called a totally geodesic submanifold.

(C) Copyright Australian Mathematical Society 1980

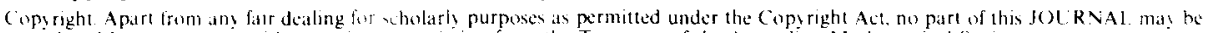
reproduced by any procesis withoul written permission from the Treasurer of the Australian Mathemidtical Sociely. 
In Wolf (1963), totally geodesic submanifolds in rank one symmetric spaces are classified. Totally geodesic submanifolds in symmetric spaces of higher ranks have been studied in Chen-Nagano $(1977,1978)$ in which the $\left(M_{+}, M_{-}\right)$-theory is introduced. In this paper, we shall generalize the results of Wolf (1963) and ChenNagano $(1977,1978)$ to totally umbilical submanifolds. In particular, we shall prove the following theorems.

THEOREM 1. If an irreducible symmetric space $M$ admits a totally umbilical hypersurface $N$, then both $M$ and $N$ are of constant curtature.

THEOREM 2. Let $N$ be a totally umbilical submanifold in a symmetric space $M$. If $\operatorname{dim} M-\operatorname{dim} N<\operatorname{rank} M-1$, then the mean curvature rector is parallel (in the normal bundle). In particular, the mean curvature is constant and $N$ is either totally geodesic or of constant curvature.

THEOREM 3. If $N$ is a totally umbilical submanifold in an irreducible symmetric space $M$, then

$$
\operatorname{codim} N \geqslant \operatorname{rank} M-1 .
$$

THEOREM 4. Let $N$ be a totally umbilical submanifold with constant mean curtature in a compact symmetric space $M$. Then

(i) either $N$ is totally geodesic or $N$ is of positive constant curvature, and

(ii) if the mean curvature vector $H$ is not parallel, and $\operatorname{dim} \mathrm{N}>2$, then $\operatorname{dim} N<\frac{1}{2} \operatorname{dim} M$.

REMARK. Totally umbilical submanifold with parallel mean curvature vector in (locally) symmetric spaces have been completely classified in Chen (1979).

For general results on symmetric spaces, see Helgason (1968).

The estimate of codimension in Theorem 2 is best possible.

\section{Preliminaries}

Let $N$ be an $n$-dimensional submanifold of a Riemannian manifold $M$ with metric $g$. For a vector field $\xi$ normal to $N$, we write

$$
\tilde{\nabla}_{X} \xi=-A_{\xi} X+D_{X} \xi
$$

where $-A_{\xi} X, D_{X} \xi$ are the tangential and normal components of $\tilde{\nabla}_{X} \xi$, respectively. A normal vector field $\xi$ is said to be parallel (in the normal bundle) if $D_{X} \xi=0$ for all $X$ tangent to $N$.

Let $R$ and $\tilde{R}$ be the curvature tensors associated with $\nabla$ and $\tilde{\nabla}$, respectively. For 
example,

$$
R(X, Y)=\nabla_{X} \nabla_{Y}-\nabla_{Y} \nabla_{X}-\nabla_{[X, Y]}
$$

A Riemannian manifold $M$ is locally symmetric if $\tilde{\nabla} \tilde{R}=0$.

For the second fundamental form $h$, we define the covariant derivative in $(T N) \oplus\left(T^{\perp} N\right)$, to be

$$
\left(\bar{\nabla}_{X} h\right)(Y, Z)=D_{X}(h(Y, Z))-h\left(\nabla_{X} Y, Z\right)-h\left(Y, \nabla_{X} Z\right),
$$

where $T N$ and $T^{\perp} N$ denote the tangent and normal bundles of $N$, respectively.

For vector fields $X, Y, Z, W$ tangent to $N$, the equations of Gauss and Codazzi take the forms:

(2.4) $R(X, Y ; Z, W)=\tilde{R}(X, Y ; Z, W)+g(h(X, W), h(Y, Z))-g(h(X, Z), h(Y, W))$,

$$
(\tilde{R}(X, Y) Z)^{\perp}=\left(\bar{\nabla}_{X} h\right)(Y, Z)-\left(\bar{\nabla}_{Y} h\right)(X, Z),
$$

where $R(X, Y ; Z, W)=g(R(X, Y) Z, W), \ldots$, etc., and $\downarrow$ in (2.5) denotes the normal component.

Let $X$ and $Y$ be two orthonormal vectors tangent to $N$. The sectional curvature $K(X \wedge Y)$ of the plane $X \wedge Y$ spanned by $\{X, Y\}$ is given by

$$
K(X \wedge Y)=R(X, Y ; Y, X) .
$$

We denote by $K$ the sectional curvature for $M$. By an extrinsic sphere we mean a totally umbilical submanifold with nonzero parallel mean curvature vector. We mention the following lemma for later use.

Lemma 1 (Chen 1979)). Let $N$ be an n-dimensional extrinsic sphere in a locally symmetric space $M$. Then $M$ admits an $(n+1)$-dimensional totally geodesic submanifold $\tilde{N}$ such that (a) $N$ is an extrinsic sphere of $\tilde{N}$ and (b) both $N$ and $\tilde{N}$ are of constant curcature.

An isometry $s$ of a Riemannian manifold is said to be involutive if its iterate $s^{2}$ is the identity map. A Riemannian manifold $M$ is a symmetric space if, for each point $p$ of $M$, there exists an involutive isometry $s_{p}$ of $M$ such that $p$ is an isolated fixed point of $s_{p}$. It is well known that every symmetric space is a complete locally symmetric space. And every locally symmetric space is locally an open submanifold of a symmetric space.

We denote by $G$ the closure of the group of isometries generated by $\left\{s_{p}: p \in M\right.$; in the compact-open topology. Then $G$ acts transitively on $M$; hence the typical isotropy subgroup $K$, say at 0 , is compact and $M=G / K$.

Let $\sigma_{0}$ be the involutive automorphism of $G$ given by $\sigma_{0}(x)=s_{0} \cdot x \cdot s_{0}, x \in G$. Then $\sigma_{0}$ fixes $K$ and it induces an involutive automorphism of the Lie algebra $g$ of $G$. The 
Cartan decomposition of $\mathfrak{g}$ is then given by

$$
\mathfrak{g}=\mathfrak{t}+\mathfrak{m},
$$

where $f$ and $m$ are the eigen spaces of $\sigma_{0}$ with eigen values 1 and -1 , respectively. It is known that $\mathfrak{f}$ is the Lie algebra of $K$ and we have

$$
[\mathfrak{l}, \mathfrak{t}] \subset \mathfrak{k}, \quad[\mathfrak{k}, \mathrm{m}] \subset \mathrm{m}, \quad[\mathrm{m}, \mathrm{m}] \subset \mathfrak{t} .
$$

Moreover, $m$ can be identified with the tangent space of $M$ at 0 .

The following lemmas of E. Cartan are well known (see Helgason (1968)).

LEMMA 2. The curvature tensor $\tilde{R}$ of $M$ at 0 satisfies

$$
\tilde{R}(\tilde{X}, \tilde{Y}) \tilde{Z}=-[[\tilde{X}, \tilde{Y}], \tilde{Z}]
$$

for $\tilde{X}, \tilde{Y}, \tilde{Z} \in \mathbf{m}$.

Lemma 3. Let $B$ be a totally geodesic submanifold of $M$ through 0 . Then $B$ is flat if and only if $[\pi, \pi]=0$ where $\pi=T_{0} B \subset T_{0} M=\mathbf{m}$.

We mention the following unpublished result of Chen-Nagano for later use. This result is proved by using the $\left(M_{+}, M_{-}\right)$-theory (Chen-Nagano (1978)).

LEMMA 4. Every totally geodesic submanifold $B$ of an ireducible locally symmetric space $M$ satisfies

$$
\operatorname{dim} B \leqslant \operatorname{dim} M-\operatorname{rank} M .
$$

\section{Proof of Theorem 1}

Let $N$ be a totally umbilical hypersurface of an irreducible symmetric space $M$. Then by (1.2) and (2.3) we have

$$
\left(\bar{\nabla}_{X} h\right)(Y, Z)=g(Y, Z) \cdot D_{X} H .
$$

Thus by (3.1) and the Codazzi equation, we find

$$
\tilde{R}(X, Y ; Z, H)=\frac{1}{2}\left\{g(Y, Z)\left(X \alpha^{2}\right)-g(X, Z)\left(Y \alpha^{2}\right)\right\},
$$

where $\alpha^{2}=g(H, H)$. Let $E_{1}, \ldots, E_{n}$ be an orthonormal basis of $T_{p} N, p \in N$. Then (3.2) implies

$$
\tilde{R}\left(E_{1}, E_{i} ; E_{i}, H\right)=\frac{1}{2}\left(E_{1} \alpha^{2}\right)
$$


for $i=2, \ldots, n$. Consequently, the Ricci tensor $\tilde{S}$ of $M$ satisfies

$$
\tilde{S}\left(E_{1}, H\right)=\frac{n-1}{2}\left(E_{1} \alpha^{2}\right) .
$$

On the other hand, since $M$ is Einsteinian, $\tilde{S}\left(E_{1}, H\right)=0$. Thus we find that $E_{1} \alpha^{2}=0$. Since $E_{1}$ can be chosen to be any unit vector, $\alpha$ is constant. Moreover, because $N$ is a hypersurface, $N$ is either an extrinsic sphere or a totally geodesic hypersurface. If $N$ is an extrinsic sphere, Lemma 1 implies that both $N$ and $M$ are of constant curvature. If $N$ is a totally geodesic hypersurface, Lemma 4 implies that $M$ is a locally symmetric space of rank one. From Wolf's result (Wolf (1963)), we know that this is impossible unless $M$ is locally isometric to a sphere, or a hyperbolic space. In both cases, $M$ has constant curvature. This proves Theorem 1.

\section{Proof of Theorems 2 and 3}

Suppose that $N$ is a totally umbilical submanifold of a symmetric space $M$. We may choose any fixed point $p$ in $M$ as the origin of $M$. For any given nonzero vector $X \in T_{p} N$, there exists a flat totally geodesic submanifold $B$ through $p$ such that $X \in T_{p} B$ and $\operatorname{dim} B=\operatorname{rank} M$. If $\operatorname{dim} M-\operatorname{dim} N<\operatorname{rank} M-1$, we have

$$
\operatorname{dim}\left(T_{p} N \cap T_{p} B\right) \geqslant 2 .
$$

Thus there is a unit vector $Y$ in $T_{p} N \cap T_{p} B$ so that $g(X, Y)=0$. Since $N$ is totally umbilical, we have

$$
(\tilde{R}(X, Y) Z)^{\perp}=g(Y, Z) \cdot D_{X} H-g(X, Z) \cdot D_{Y} H .
$$

Consequently, we obtain

$$
(\tilde{R}(X, Y) Y)^{\perp}=D_{X} H .
$$

On the other hand, since $B$ is a flat totally geodesic submanifold of $M$, Lemma 3 says that the tangent space $T_{p} B$ forms an abelian linear subspace of $m$. In particular, we have $[X, Y]=0$. Substituting this into (2.8) we find

$$
\tilde{R}(X, Y) Y=0 .
$$

Combining (4.3) and (4.4) we find $D_{X} H=0$. Since $X$ can be chosen to be any vector tangent to $N$ at $p$ and $p$ can be chosen to be any point in $N$, the mean curvature vector $H$ is parallel in the normal bundle. In particular, the mean curvature is constant and from Lemma 1, we see that $N$ is either totally geodesic or of constant curvature. This proves Theorem 2.

If $M$ is an irreducible symmetric space, Lemma 4 shows that $M$ admits no totally geodesic submanifold of codimension < rank $M$. From the discussion above, we see 
that every totally umbilical submanifold of codimension $<\operatorname{rank} M-1$ is an extrinsic sphere. Therefore, by using Lemma $1, M$ admits a totally geodesic submanifold of codimension < rank $M-2$. This contradicts to Lemma 4 again. Theorem 3 is thus proved.

\section{Proof of Theorem 4}

Let $N$ be a totally umbilical submanifold with constant mean curvature in a symmetric space. Then, by a result of Miyazawa and Chüman (1972), $N$ is either totally geodesic or a locally symmetric space with vanishing Weyl conformal curvature tensor. In the following, we assume that $N$ is not totally geodesic.

Case (a). If $N$ is irreducible and locally symmetric, $N$ is Einsteinian. Since every Einstein manifold with vanishing Weyl conformal curvature tensor is of constant curvature, $N$ is of constant curvature.

Case (b). If $N$ is reducible and of dimension 2 or 3 , then $N$ is locally the product of two locally symmetric spaces of dimension $\leqslant 2$. Since every locally symmetric space of dimension 2 is of constant curvature, $N$ is either flat or a local product of a curve and a surface of constant curvature.

Case (c). If $N$ is reducible and of dimension $\geqslant 4$, then from Proposition 2 of Goldberg (1969), we see that $N$ is a flat space, a local product of two spaces of constant curvatures $c$ and $-c$, or a local product of a curve and a space of constant curvature.

Consequently, if $N$ is not totally geodesic, $N$ is one of the following spaces: (1) a space of constant curvature, (2) a local product of two spaces of constant curvatures $c$ and $-c$, respectively, or (3) a local product of a curve and a space of constant curvature.

Now, if $M$ is a compact symmetric space, $M$ is always nonnegatively curved. Thus, by the equation of Gauss, if $N$ is not totally geodesic, $N$ is always positively curved. The cases (2) and (3) cannot occur. Therefore, $N$ is either totally geodesic or of positive constant curvature. This proves part (i) of Theorem 4.

Now, suppose that $\operatorname{dim} N>2$ and the mean curvature vector is not parallel. From part (i), $N$ is of positive constant curvature $c$. Since $N$ is a totally umbilical submanifold of constant mean curvature, (3.2) gives

$$
\tilde{R}(X, Y ; Z, H)=0
$$

for vector fields $X, Y, Z$ tangent to $N$. By using $\tilde{\nabla} \tilde{R}=0,(1.1),(1.2),(2.5)$ and (5.1) we 
find

$$
\begin{aligned}
& x^{2} \tilde{R}(X, Y ; Z, U)=g(U, X) \tilde{R}(H, Y ; Z, H)-g(U, Y) \tilde{R}(H, X ; Z, H) \\
&+ g(Y, Z) g\left(D_{X} H, D_{U} H\right)-g(X, Z) g\left(D_{Y} H, D_{U} H\right),
\end{aligned}
$$

for $U$ tangent to $N$. Let $X=U, Y=Z$ be orthonormal vectors tangent to $N$. Then (2.4) and (5.2) gives

$$
\alpha^{2} \tilde{K}(H \wedge Y)=-\left|D_{X} H\right|^{2}+\alpha^{2} c-\alpha^{4},
$$

where $c$ is the constant sectional curvature of $N$.

Since this is true for all orthonormal vectors $X$ and $Y$ tangent to $N$ and $\operatorname{dim} N>2$, $\left|D_{X} H\right|$ is independent of $X$. In particular, since $H$ is not parallel, $\left|D_{X} H\right|$ is nonzero for any unit vector $X$ tangent to $N$ at some point $p$ in $N$.

Let $U=X=E_{i}$ and summing on $i$ in (5.2) we find

$$
\begin{aligned}
(n-1) \tilde{R}(H, Y ; Z, H)=\alpha^{2} S(Y, Z)-(n-1) \alpha^{4} g(Y, Z) \\
\\
\quad+g\left(D_{Y} H, D_{Z} H\right)-g(Y, Z)|D H|^{2},
\end{aligned}
$$

by virtue of (2.4), where $|D H|^{2}=\sum_{i=1}^{n} g\left(D_{E_{i}} H, D_{E_{i}} H\right)$ and $S$ is the Ricci tensor of $N$.

Substituting (5.4) into (5.2) we obtain

$$
\begin{aligned}
& (n-1) x^{2} R(X, Y ; Z . U) \\
& =|D H|^{2}\{g(Y, U) g(X, Z)-g(X, U) g(Y, Z)\} \\
& +g(U, X)\left\{\alpha^{2} S(Y, Z)+g\left(D_{Y} H, D_{Z} H\right)\right\} \\
& -g(U, Y)\left\{\alpha^{2} S(X, Z)+g\left(D_{X} H, D_{Z} H\right)\right\} \\
& +(n-1)\left\{g(Y, Z) g\left(D_{X} H, D_{U} H\right)-g(X, Z) g\left(D_{Y} H, D_{U} H\right)\right\}
\end{aligned}
$$

By setting $Y=Z=E_{\mathrm{i}}$ and summing on $i$, we get

(5.6) $(n-2) g\left(D_{X} H, D_{\imath} H\right)=\alpha^{2} S(X, U)+\frac{n-2}{n} g(X, U)|D H|^{2}-\frac{1}{n} \alpha^{2} \tau g(X, U)$,

where $\tau=\sum_{i=1}^{n} S\left(E_{i}, E_{i}\right)$ is the scalar curvature of $N$.

Since $N$ is of constant curvature, (5.6) shows that

$$
g\left(D_{X} H, D_{U} H\right)=0
$$

for orthonormal vectors $X$ and $U$. Because $D_{X} H$ is nonzero at $p$ for all unit vector $X \in T_{p} N$. (5.7) tells us that

$$
H, D_{E_{1}} H, \ldots, D_{E_{n}} H
$$

are mutually orthogonal and they span an $(n+1)$-dimensional linear subspace of the normal space $T_{p}^{\perp} N$. This proves part (ii) of Theorem 4 . 


\section{References}

B. Y. Chen (1979), 'Extrinsic spheres in Riemannian manifolds', Houston J. Math. 5, 319-324.

B. Y. Chen and T. Nagano (1977), 'Totally geodesic submanifolds of symmetric spaces, I', Duke. Math. J. 44. 745-755.

B. Y. Chen and T. Nagano (1978), 'Totally geodesic submanifolds of symmetric spaces, II', Duke Math.J. 45, 405-425.

S. I. Goldberg (1969), 'On conformally flat spaces with definite Ricci curvature', Kodai Math. Se'm. Re'p. 21. 226-232.

S. Helgason (1968), Differential geometry, Lie groups and symmetric spac's (Academic Press, New York).

T. Miyazawa and G. Chūman (1972), On certain subspaces of Riemannian recurrent spaces:. Tensor 23. 253-260.

J. A. Wolf (1963), 'Elliptic spaces in Grassmann manifolds', Illinois J. Math. 7, 447-462.

\section{Department of Mathematics}

Michigan State University

East Lansing, Michigan 48824

U.S.A. 